\title{
Quadrature Methods Based on Complex Function Values*
}

\author{
By J. N. Lyness
}

\begin{abstract}
A method of numerical quadrature over a finite interval is described. This method is applicable if the integrand is an analytic function, regular within the circle in the complex plane having the integration interval as diameter. The method is iterative in nature and relies on function values at equally spaced points on this circle. It is flexible enough to take into account certain simple nonanalytic singularities in the integrand lying on the interval of integration or its extension.

Numerical examples are given which illustrate various advantages and disadvantages of this method when compared with standard quadrature procedures.
\end{abstract}

1. Introduction. In many scientific and engineering problems, the mathematical equations which have to be solved are so difficult or involved that no simple analytical solution is known. In these cases, a familiar approximation consists of replacing some function $f(x)$ by the first several terms of its Taylor expansion about some convenient point $x_{0}$. This type of approximation, carried out analytically, may lead to an approximation to the solution of the problem, which if not exact, hopefully retains the physical features of interest. Occasionally the error incurred by making such an approximation can be bounded or estimated approximately.

In the procedures of computational mathematics, this approach to a problem is not common. A truncated Taylor series may, naturally, be used in order to construct the numerical procedure. But the procedure itself is expressed in terms of other quantities, such as function values at neighboring points. It is very uncommon to make explicit use of a Taylor expansion. There are usually several sound reasons for avoiding this, but in the absence of any other reason there is the well-known phenomenon that the numerical calculation of derivatives in terms of neighboring function values is a process subject to large and unpredictable error due to the amplification of round-off error.

However, if the function in question is an analytic function and there exists a Taylor series

$$
f\left(x_{0}+z\right)=a_{0}+a_{1} z+a_{2} z^{2}+\cdots, \quad\left|z-x_{0}\right|<R_{c},
$$

whose circle of convergence includes the region of interest for the particular problem, this specific difficulty may be circumvented. This is accomplished by taking advantage of Cauchy's expression for the derivative

$$
a_{s}=\frac{f^{(s)}\left(x_{0}\right)}{s !}=-\frac{1}{2 \pi i} \oint_{c} \frac{f(z)}{\left(z-x_{0}\right)^{s+1}} d z
$$

and evaluating a contour integral numerically. In this way the conventional procedure for numerical differentiation, which is based on high-order differences, is

Received September 26, 1968.

* Work performed under the auspices of the U. S. Atomic Energy Commission. 
replaced by one based on techniques of numerical quadrature. There is, of course, round-off error involved, but this is predictable and may be controlled by means of choosing a suitable contour $C$. However, the process does require function values in the complex plane and is obviously restricted to analytic functions.

The idea of basing a numerical quadrature rule for a real-valued function on function values $f\left(z_{i}\right)$ where $z_{i}$ is complex is not new. In 1950 Birkhoff and Young [3] published a 'five point formula'

$$
\int_{-1}^{1} f(z) d z \simeq \frac{1}{15}\{24 f(0)+4(f(1)+f(-1))-(f(i)+f(-i))\}
$$

which is exact when $f(x)$ is a polynomial of degree 5 or less. Later on McNamee [9] considered methods of calculating certain types of infinite integrals by carrying out numerical contour integration of related functions on different contours.

In this paper we discuss numerical procedures for various quadrature problems. These are based on the prior calculation of Taylor coefficients using complex function values (described in Section 2) and consequent term by term integration of the Taylor series. In Section 3 we deal specifically with the definite integral

$$
\int_{-r}^{r} f(x) d x=2 r \sum_{s=0 ;}^{\infty} \frac{r^{s} a_{s}}{s+1} .
$$

An iterative quadrature scheme is presented and is compared with standard quadrature methods including the Gauss-Legendre formulas, Romberg integration and the Adaptive Simpson Technique. In subsequent sections we discuss more briefly the application to indefinite quadrature; for example

$$
\int_{-r}^{B_{i}} f(x) d x=F\left(B_{i}\right)-F(-r),
$$

where the value of the integral is required for several values of $B_{i}$. We also deal with quadratures whose integrand has a singularity of the type $\left(x-x_{0}\right)^{n} \ln \left|x-x_{0}\right|$ or $\left|x-x_{0}\right|^{\alpha}$ either within the interval of integration or close to it on the real axis. In this type of application the method shows up extremely well. Finally in Section 6 we mention briefly a method of interval subdivision which has to be applied if the integrand has singularities in the complex plane close to the interval of integration.

We should perhaps emphasize that, although complex function values are used, we are interested particularly in the quadrature of a function $f(x)$ which is real on the real line. The use of complex variables is merely a tool which ultimately produces a real result. However, the methods given here can be applied to complex integrands directly.

2. The Taylor Coefficients. In this section we deal exclusively with a method, based on function evaluation, for the construction of a set of approximations $a_{s}{ }^{(m)}$ to the Taylor coefficients $a_{s}$ of an analytic function $f(z)$. This has a Taylor series expansion about the origin

$$
f(z)=a_{0}+a_{1} z+a_{2} z^{2}+\cdots \quad|z|<R_{c},
$$

the radius of convergence being $R_{c}$ (or being infinite). In this process we calculate approximations to $r^{s} a_{s}$ which we term 'normalized Taylor coefficients.' Here 


$$
0<r<R<R_{c},
$$

$R$ being an intermediate radius of which we occasionally make use. It is convenient to refer to the circle whose center is the origin and whose radius is $r, R$, or $R_{c}$, by $C_{r}, C_{R}$, or $C_{R_{c}}$ respectively. This section is conveniently divided into three parts. In the first part we derive the relevant formula, in the second we describe a convenient iteration process and in the third we discuss the convergence, the stopping criterion for the iteration process and the effect and control of round-off error.

2(i). Formulas for the Taylor Coefficients. The results of this subsection are well known. Useful discussion of various aspects of these results occur, for example in Krylov [13, p. 74] and in Davis and Rabinowitz [12, pp. 53-58]. The very brief derivation is given here in order to establish the notation and to include appropriate background material for subsequent results.

If we apply Cauchy's theorem for the derivative, we find

$$
r^{s} a_{s}=\frac{r^{s} f^{(s)}(0)}{s !}=\frac{r^{s}}{2 \pi i} \int_{c_{r}} \frac{f(z)}{z^{s+1}} d z=\int_{0}^{1} g_{s}(t) d t, \quad s=0,1,2, \cdots
$$

where we have written

$$
g_{s}(t)=e^{-2 \pi i s t} f\left(r e^{2 \pi i t}\right) .
$$

This function is a complex-valued analytic function of real variable $t$ and is periodic having period 1. A Fourier expansion may be obtained by substituting (2.1) into (2.4) to give

$$
g_{s}(t)=\sum_{k=0}^{\infty} e^{2 \pi i k t}\left(r^{k+s} a_{k+s}\right) .
$$

Consequently $g_{s}(t)$ has Fourier coefficients which correspond to the normalized Taylor coefficients of $f^{(s)}(z)$.

Since $g_{s}(t)$ has a period equal to the interval of integration, a convenient quadrature rule to use for the evaluation of the integral in (2.3) is the trapezoidal rule $R^{[m, 1]} g$, defined by

$$
R^{[m, 1]} g=\frac{1}{m}\left\{\sum_{j=1}^{m-1} g\left(\frac{j}{m}\right)+\frac{1}{2}(g(0)+g(1))\right\} .
$$

The resulting approximation to $r^{s} a_{s}$ is denoted by $r^{s} a_{s}{ }^{(m)}$, defined by

$$
r^{s} a_{s}^{(m)}=R^{[m, 1]} g_{s} \text {. }
$$

This approximation requires $m$ function values for its evaluation.

It is well known that an $m$-interval trapezoidal rule integrates exactly any trigonometric polynomial of degree $m-1$ or less. In particular

$$
\begin{aligned}
& R^{[m, 1]}\left(e^{2 \pi i k x}\right)=0, \quad|k / m| \neq \text { integer or zero }, \\
& R^{[m, 1]}\left(e^{2 \pi i k x}\right)=1, \quad|k / m|=\text { integer or zero } .
\end{aligned}
$$

Applying this result separately to each term in (2.5) we find

$$
r^{s} a_{s}^{(m)}=R^{[m, 1]} g_{s}=r^{s} a_{s}+r^{s+m} a_{s+m}+r^{s+2 m} a_{s+2 m}+\cdots \quad m>s,
$$


and this series is convergent since the Taylor series (2.1) has a radius of convergence which exceeds $r$.

Equation (2.10) gives us the polynomial degree of the approximation $r^{8} a_{s}{ }^{(m)}$ to the exact value $r^{s} a_{s}$. If $f(z)$ is a polynomial of degree $s+m-1$, the Taylor coefficients $a_{i}(i \geqq s+m)$ are identically zero and (2.10) reduces to the exact equality

$$
r^{8} a_{s}{ }^{(m)}=r^{s} a_{s} .
$$

Consequently we may state

THEOREM 2.12. The set of approximations

$$
r^{s} a_{s} \simeq r^{s} a_{s}^{(m)}=\frac{1}{m} \sum_{j=1}^{m} e^{-2 \pi i j s / m} f\left(r e^{2 \pi i j / m}\right) \quad s=0,1,2, \cdots, m-1
$$

are exact if $f(z)$ is a polynomial of degree $m-1$ or less.

We note that this set of $m$ quantities are simple combinations of $m$ function values $f\left(z_{i}\right)$ where $z_{i}$ are equally spaced on the circle $C_{r}:|z|=r$ and $m$ constants $e^{-2 \pi i j / m}(j=1, \cdots, m)$ equally spaced on the unit circle. Consequently the calculation of this set of approximation requires $m$ calls to a complex exponential routine (perhaps replaced by a data statement). Once this is accomplished, these $m$ numbers act both as abscissas for $m$ calls to the function routine, and in a different order as weights. The coding is mainly a matter of bookkeeping-to see that the correct coefficient is attached to the appropriate function value in the calculation of a particular Taylor coefficient.

2(ii). An Iteration Procedure. The formulas (2.12) given above were derived by applying the endpoint trapezoidal rule $R^{[m, 1]} g$ given by (2.6) to the contour integral expression (2.3) for the sth derivative. One may obtain a large number of similar formulas simply by applying different quadrature rules to the same integral. One such rule is convenient from the point of view of describing an iterative procedure. This is the midpoint trapezoidal rule

$$
R^{[m, 0]} g=\frac{1}{m} \sum_{j=1}^{m} g\left(\frac{j-\frac{1}{2}}{m}\right) .
$$

The theory given in subsection 2(i) applies to this approximation almost unaltered. The only modifications required are phase factors +1 or -1 multiplying individual terms in some of the expansions. The result is a theorem corresponding to Theorem 2.12 , namely

Theorem 2.14. The set of approximations

$$
\begin{aligned}
r^{s} a_{s} \simeq r^{s} b_{s}{ }^{(m)}=\frac{1}{m} \sum_{j=1}^{m} e^{-2 \pi i(2 j-1) s / 2 m} f\left(r e^{2 \pi i(2 j-1) / 2 m}\right) & \\
s & =0,1,2, \cdots, m-1
\end{aligned}
$$

are exact if $f(z)$ is a polynomial of degree $m-1$ or less.

This set of approximations is based on a different set of function values. In fact, by inspection it is apparent that the set required to calculate $r^{s} b_{s}{ }^{(m)}$, together with the set required to calculate $r^{s} a_{s}{ }^{(m)}$ form together the set required to calculate $r_{s} a_{s}^{(2 m)}$. This circumstance may be exploited to construct an iteration procedure.

We note from (2.12) and (2.14) that 


$$
\begin{aligned}
& r^{s} a_{s+m}^{(m)}=r^{s} a_{s}^{(m)}, \quad s=0,1, \cdots, m-1, \\
& r^{s} b_{s+m}^{(m)}=:-r^{s} b_{s}^{(m)}, \quad s=0,1, \cdots, m-1,
\end{aligned}
$$

and that in general

$$
R^{[2 m, 1]} g \equiv \frac{1}{2}\left\{R^{[m, 1]} g+R^{[m, 0]} g\right\} .
$$

These relations may be used to establish

$$
\begin{aligned}
& r^{s} a_{s}^{(2 m)}=\frac{1}{2}\left\{r^{s} a_{s}^{(m)}+r^{s} b_{s}^{(m)}\right\}, \quad s=0,1, \cdots, m-1, \\
& r^{s} a_{s+m}^{(2 m)}=\frac{1}{2}\left\{r^{s} a_{s}^{(m)}-r^{s} b_{s}^{(m)}\right\}, \quad s=0,1, \cdots, m-1,
\end{aligned}
$$

and these formulas may be used as the basis of the iteration procedure described here. This starts with the very poor approximation

$$
a_{0}^{(1)}=f(r)
$$

and the first stage consists simply of the calculations

$$
\begin{aligned}
b_{0}{ }^{(1)} & =f(-r), \\
a_{0}{ }^{(2)} & =\frac{1}{2}\left(a_{0}{ }^{(1)}+b_{0}{ }^{(1)}\right)=\frac{1}{2}(f(r)+f(-r)), \\
r a_{1}{ }^{(2)} & =\frac{1}{2}\left(a_{0}{ }^{(1)}-b_{0}{ }^{(1)}\right)=\frac{1}{2}(f(r)-f(-r)),
\end{aligned}
$$

leaving us with the values of $a_{0}{ }^{(2)}$ and $r a_{1}{ }^{(2)}$. The general stage starts with a set of normalized Taylor coefficients $r^{s} a_{s}{ }^{(m)}(s=0,1, \cdots, m-1)$ where $m$ is a power of two. Using (2.14), which requires $m$ function evaluations, we calculate the set $r^{s} b_{s}{ }^{(m)}(s=0,1, \cdots, m-1)$. Then using (2.18) and (2.19) we calculate the set $r^{s} a_{s}{ }^{(2 m)}(s=0,1, \cdots, 2 m-1)$. This completes this stage. It is worth noting that once the set $r^{s} b_{s}{ }^{(m)}$ has been calculated the function evaluations required for this calculation are not required again. Also once the set $r^{s} a_{s}{ }^{(2 m)}$ has been calculated, the previous sets $r^{s} a_{s}{ }^{(m)}$ and $r^{s} b_{s}{ }^{(m)}$ are not required again.

This iteration procedure has the property that each stage requires twice as many function evaluations as the previous stage. The effort in computer time required in one stage is slightly greater than the effort required in all the previous stages added together. Consequently if we fail to terminate the process when sufficient accuracy has been achieved, but carry out a single unnecessary iteration, the effort required for the whole calculation is doubled. (Incidentally the effect on the accuracy of the result is that the number of correct significant figures is also approximately doubled.) It is important to have available a criterion for gauging the accuracy at any stage, so that the iteration may be terminated appropriately.

In the applications described in Section 3, only the even Taylor coefficients are required, and in other applications only the odd Taylor coefficients are required. Unless $m=1$, the relations (2.18) and (2.19) indicate that a particular approximation $a_{s}{ }^{(2 m)} s$ odd (even) depends only on previous approximations $a_{s}^{(m)}, b_{s}{ }^{(m)} s$ odd (even). Thus a nontrivial amount of calculation may be avoided by simply omitting the calculation of any odd (even) approximation $a_{s}{ }^{(m)} s$ odd (even), except at the first stage. However, the same number of function evaluations are required to 
obtain a particular accuracy, whether or not some of the Taylor coefficients are not needed.

2(iii). Convergence of Iteration Process. The convergence of this process may be demonstrated by means of Cauchy's inequality for the $n$th derivative. This inequality may be written in the form

$$
\left|r^{j} a_{j}\right|^{2} \leqq I(R) \rho^{2 j}
$$

where $R$ is the radius of $C_{R}$ and satisfies

$$
r<R<R_{c}
$$

and

$$
\rho=r / R<1
$$

and

$$
I(R)=\frac{1}{2 \pi i} \int_{C_{R}}|f(z)|^{2} \frac{d z}{z}=\left.\int_{0}^{1} f\left(R e^{2 \pi i t}\right)\right|^{2} d t .
$$

Equation (2.10) gives the exact error

$$
r_{s} a_{s}^{(m)}-r_{s} a_{s}=\sum_{k=1}^{\infty} r^{s+k m} a_{s+k m} .
$$

A bound on the magnitude of the error is obtained by replacing each term on the right of this expression by the bound given by (2.23). This gives

$$
\left|r^{*} a_{s}^{(m)}-r^{s} a_{s}\right|<\frac{(I(R))^{1 / 2} \rho^{s+m}}{1-\rho^{m}} .
$$

(A slightly closer bound in which $\left(1-\rho^{2 m}\right)^{-1 / 2}$ replaces $\left(1-\rho^{m}\right)^{-1}$ may be derived by other methods.) Since $\rho<1$, it follows immediately that

$$
\operatorname{Lim}_{m \rightarrow \infty}\left|r^{s} a_{s}^{(m)}-r^{s} a_{s}\right|=0 \text {. }
$$

We turn now to the question of deciding when to terminate the iteration. This is a practical question and depends to some extent on the use to which the Taylor coefficients, once calculated, are to be put.

It is pertinent to point out here that one of the quantities being calculated, namely $a_{0}$, may be independently determined by a single function evaluation $f(0)$. Thus

$$
\left|a_{0}{ }^{(m)}-f(0)\right|<\frac{(I(R))^{1 / 2} \rho^{m}}{1-\rho^{m}}=E_{0}{ }^{(m)} ;
$$

moreover the corresponding bounds on the other normalized Taylor coefficients are stronger than this

$$
\left|r^{s} a_{s}^{(m)}-r^{s} a_{0}\right|<E_{0}^{(m)} \rho^{s} .
$$

A plausible, but nonrigorous, procedure is to terminate the calculation when $\left|a_{0}{ }^{(m)}-f(0)\right|<\epsilon_{\text {tol }}$, some given tolerance level.

It is dangerous to use this criterion without modification since it may happen by 
chance that $a_{0}{ }^{(m)}$ is exceptionally close to $f(0)$ for some value of $m$. To guard against this hazard, as a practical procedure, the following precaution has been used.

After each stage in the iteration, we may note the value

$$
b_{n}=\left|f(0)-a_{0}{ }^{\left(2^{n}\right)}\right|
$$

and we may extrapolate to form estimates of $b_{n}$, based on values of $b_{i}, i<n$, assuming a functional relationship

$$
b_{n}=K A^{2^{n}} .
$$

For example

$$
b_{n}^{(1,2)}=b_{n-1}^{3} / b_{n-2}^{2}
$$

is the value of $b_{n}$ we should expect if (2.33) were satisfied by all $b_{n}$. Similarly

$$
b_{n}{ }^{(1,3)}=\left(b_{n-1}^{7} / b_{n-3}^{4}\right)^{1 / 3} .
$$

In a code written by the author, the convergence criterion was based on

$$
\epsilon_{\mathrm{est}}^{\left(2^{n}\right)}=\max \left(b_{n}, \min \left(b_{n-1}, b_{n}{ }^{(1,2)}, b_{n}{ }^{(1,3)}\right)\right)
$$

and convergence was allowed if $\epsilon_{\mathrm{est}}^{(m)}<\epsilon_{\mathrm{tol}}$. In this way some additional indication that the general error was in fact less than $\epsilon_{\text {tol }}$ was required. This choice is quite heuristic and the user might apply much more stringent criteria.

This convergence criterion cannot be used if $f(z)$ is an odd function of $z$. In this case

$$
f(z)=-f(-z)
$$

and $a_{0}{ }^{(m)}$ is zero for all even $m$. Here a possible procedure is to recast the problem so that the function $f(z) / z$ is being treated, or to base the convergence upon the values $\left|r a_{1}{ }^{(m)}-r f^{\prime}(0)\right|$. In either case the value of $f^{\prime}(0)$ has to be calculated analytically. In the principal application (Section 3 ) this problem does not arise.

Finally the effect of round-off error should be estimated at each stage. A relatively coarse estimate is

$$
\epsilon_{\mathrm{r} \cdot \mathrm{o}}^{(m)}=K_{f} \epsilon_{m} \max _{i=1, m}\left|f\left(z_{i}\right)\right|,
$$

where $\epsilon_{m}$ is the machine accuracy parameter. $K_{f}$ is a factor greater than 1 provided by the user. This factor takes into account a 'safety margin' and possible inaccuracies in the function subroutine. As more and more function evaluations take place, $\epsilon_{\mathrm{r} \cdot \mathrm{o}}^{(m)}$ is updated. If at any stage

$$
\epsilon_{\text {tol }}<\epsilon_{\mathrm{r} \cdot \mathrm{o}}^{(m)}
$$

the user cannot expect to attain the required accuracy $\epsilon_{\text {tol }}$ because of round-off error. Also, if at any stage

$$
\epsilon_{\mathrm{est}}<\epsilon_{\mathrm{r} \cdot \mathrm{o}}^{(m)}
$$

this estimate is not reliable and has to be replaced by

$$
\tilde{\epsilon}_{\mathrm{est}}=\max \left(\epsilon_{\mathrm{est}}, \epsilon_{\mathrm{r} \cdot \mathrm{o}}^{(m)}\right) \text {. }
$$


Further calculation is then of very doubtful value. An encouraging aspect of this round-off error calculation is that in general $\epsilon_{\mathrm{r} \cdot \mathrm{o}}^{(m)}$ becomes close to its maximum for a small value of $m$. A calculation which could, in the absence of round-off error, require 64 function evaluations, might be abandoned at a relatively early stage, perhaps after only the first four or eight function evaluations, because of round-off error. This early recognition of the presence of round-off error is a most encouraging feature of this type of procedure.

3. Iterative Quadrature Scheme. In this section we discuss an application of the calculation of Taylor coefficients to the evaluation of

$$
I f=\int_{-r}^{r} f(x) d x=2 r \sum_{s=0 ; s \text { even }}^{\infty} \frac{r^{s} a_{s}}{s+1}
$$

where $f(z)$ is an analytic function having a Taylor expansion

$$
f(z)=a_{0}+a_{1} z+a_{2} z^{2}+\cdots \quad|z|<R_{c}
$$

whose radius of convergence $R_{c}$ exceeds $r$. As described in the previous section, we may at a cost of $m$ function evaluations calculate approximations $r^{s} a_{s}{ }^{(m)}$ to the normalized Taylor coefficients $r^{s} a_{s}$ using Eq. (2.12). These approximations may be used in turn to calculate an approximation

$$
Q^{(m)} f=2 r \sum_{s=0 ;}^{m-1} \frac{r^{s} a_{s}^{(m)}}{s+1}
$$

to the exact integral If given by (3.1). If we use the iteration process of the previous section, the set of Taylor coefficient approximations $r^{s} a_{s}{ }^{(m)}, s=0,1, \cdots, m-1$ is calculated for $m=1,2,4,8, \cdots$. This procedure can be employed to calculate successive approximations $Q^{(m)} f, m=1,2,4,8, \cdots$ to the exact integral $I f$. The discretization error $I f-Q^{(m)} f$ may be written

$$
I f-Q^{(m)} f=2 r\left\{\sum_{s=0 ; s \text { even }}^{m-1} \frac{r^{s} a_{s}-r^{s} a_{s}{ }^{(m)}}{s+1}+\sum_{s=m ; s \text { even }}^{\infty} \frac{r^{s} a_{s}}{s+1}\right\} .
$$

If $f(z)$ is a polynomial of degree $m-1$ or less, it follows that $a_{s}=0(s \geqq m)$ and, from Theorem 2.12, $r_{s} a_{s}-r^{s} a_{s}{ }^{(m)}=0(s<m)$. Thus each term on the right-hand side of (3.4) is separately zero. Consequently

Theorem 3.5. The approximation

$$
I f \simeq Q^{(m)} f=2 r \sum_{s=0 ;}^{m-1} \frac{r^{s} a_{s}{ }^{(m)}}{s+1}
$$

is exact if $f(z)$ is a polynomial of degree $m-1$ or less.

We now proceed to determine an error bound corresponding to (2.28). Using (2.10) we may express (3.4) in the form

$$
I f-Q^{(m)} f=2 r \sum_{s=m ; s \text { even }}^{\infty} r^{s} a_{s}\left\{\frac{1}{s+1}-\frac{1}{s(\bmod m)+1}\right\}
$$

where $s(\bmod m)$ is as usual the smallest nonnegative integer of the set $s, s-m$, $s-2 m, \cdots$. If we use the generally rather generous inequality 


$$
\left|\frac{1}{s+1}-\frac{1}{s(\bmod m)+1}\right|<1, \quad s \geqq m
$$

and Cauchy's inequality (2.23), we find

$$
\left|I f-Q^{(m)} f\right|<\frac{2 r(I(R))^{1 / 2}}{1-\rho^{2}} \rho^{m}
$$

where as before

$$
\rho=r / R<1 ; \quad R<R_{c}
$$

and $I(R)$ is given by (2.26). This establishes the convergence of the process, i.e.

Theorem 3.10.

$$
\operatorname{Lim}_{m \rightarrow \infty} Q^{(m)} f=I f .
$$

We mention here a trivial modification of the rule $Q^{(m)} f$, by use of which the polynomial degree of approximation may be increased by 2 at the cost of one additional function evaluation. One of the Taylor coefficients $r^{s} a_{s}$ has the exact value

$$
r^{0} a_{0}=f(0) .
$$

In the case $s=0$, Eq. (2.10) has the form

$$
r^{0} a_{0}^{(m)}=f(0)+r^{m} a_{m}+r^{2 m} a_{2 m}+\cdots .
$$

Consequently if we evaluate $f(0)$ in addition to the $m$ values of $f(z)$ on the circle $C_{r}:|z|=r$, we may use this information to obtain an approximation to $r^{m} a_{m}$, i.e.,

$$
r^{m} a_{m} \simeq r^{0} a_{0}{ }^{(m)}-f(0) .
$$

This approximation is of polynomial degree $2 m-1$. Thus a modified rule $\bar{Q}^{(m)} f$ which requires $m+1$ function evaluations and is of polynomial degree $m+1$ is

$$
\bar{Q}^{(m)} f=2 r\left\{f(0)+\sum_{j=2 ;}^{m-2} \frac{r^{j} a_{j}{ }^{(m)}}{j+1}+\frac{r^{0} a_{0}{ }^{(m)}-f(0)}{m+1}\right\} .
$$

Moreover

$$
\bar{Q}^{(m)} f-Q^{(m)} f=\frac{2 r m}{m+1}\left(f(0)-a_{0}^{(m)}\right) .
$$

The five-point formula of Birkhoff and Young mentioned in the introduction (1.3) is in this notation $\vec{Q}^{(4)} f$. Other modifications of the same type are possible in cases in which even order derivatives at $z=0$ are known.

While this additional function evaluation $f(0)$ may be used to increase marginally the degree of the approximation, it may also be used to good effect as a convergence criterion. If the accuracy required is $\epsilon_{\text {tol }}$ and if

$$
\left|f(0)-a_{0}{ }^{(m)}\right| \leqq \epsilon_{\text {tol }} / 2 r
$$

the situation is that two different estimates of $I f$, based on different quadrature rules $Q^{(m)} f$ and $\bar{Q}^{(m)} f$ of different degree agree to within the required tolerance. Practical convergence criteria of this type are not uncommon in quadrature routines. However, as mentioned in subsection 2(iii) there is the chance that for a particular 
value of $m$, the expression $\left|f(0)-a_{0}{ }^{(m)}\right|$ may be very small or zero although in general practical convergence has not been attained. Consequently the practical convergence criterion should be modified along the lines suggested in subsection 2(iii).

The use of this criterion is made more plausible by the similarity of the error bounds

$$
\begin{gathered}
\left|I f-Q^{(m)} f\right|<\frac{2 r(I(R))^{1 / 2}}{1-\rho^{2}} \rho^{m}, \\
2 r\left|f(0)-a_{0}{ }^{(m)}\right|<\frac{2 r(I(R))^{1 / 2}}{\left(1-\rho^{2 m}\right)^{1 / 2}} \rho^{m} .
\end{gathered}
$$

We now give a comparison of the quadrature rule $Q^{(m)} f$ introduced here with the conventional $n$-point Gauss-Legendre rule $G_{n}$ of degree $2 n-1$. Only the roughest approximation theory is sufficient to show that the Gauss-Legendre rules are much more accurate. It is convenient to compare $Q^{(m)} f$ with $G_{m / 2} f$. We find

$$
Q^{(m)} f-I f \sim \frac{2 f^{(m)}(0)}{m !} \frac{m}{m+1} r^{m+1}+O\left(r^{m+3}\right)
$$

and

$$
G_{m / 2} f-I f \sim \frac{2^{m+1}((m / 2) !)^{4} f^{(m)}(0)}{(m+1)(m !)^{3}} r^{m+1}+O\left(r^{m+1}\right) .
$$

The ratio of the coefficients of $f^{(m)}(0)$ may be estimated using Stirling's approximation. This ratio is $2^{m}$. Thus the Gauss-Legendre formula requiring only $m / 2$ points gives a result whose error is $2^{-m}$ times the error obtained using the $m$-point formula $Q^{(m)} f$ given here. We should note that $Q^{(m)} f$ requires function evaluations at conjugate points. Thus if $f(x)$ is real $Q^{(m)} f$ requires essentially only $m / 2-1$ complex evaluations and two real evaluations, whereas $G_{m / 2} f$ requires $m / 2$ real evaluations.

The conclusion that the rule given here, taken in isolation, is considerably inferior to the Gauss-Legendre rule is quite inescapable. This comparison is valid in the case in which the user is permitted a fixed amount of work (or time on a computer) and is asked to obtain as close an approximation as possible to the exact integral. Under these circumstances, the appropriate Gauss-Legendre formula is clearly more suitable.

However, the usual problem is different. The user is permitted a fixed error $\epsilon_{\mathrm{tol}}$ in his result and is asked to obtain an approximation to this accuracy. Under these circumstances an iterative process such as that described here has certain advantages not enjoyed by the Gauss-Legendre formulas. The method suggested by Stroud [11] for using these formulas is to calculate successively $G_{4} f, G_{6} f, G_{8} f \cdots$ and to accept $G_{m} f$ if $\left|G_{m} f-G_{m-2} f\right|<\epsilon_{\text {tol }}$. This procedure is very wasteful on two separate counts. The first is that since $G_{4} f, G_{6} f, \cdots$ have no points in common, the majority of the work is not used in the final approximation at all. This feature is not common in quadrature routines. The second count is that in practice $G_{m-2} f$ is an adequate approximation which is rejected simply because $G_{m-4} f$ is so inadequate that $\left|G_{m-2} f-G_{m-4} f\right|>\epsilon_{\text {to }}$. This feature is very common in quadrature routines. A very general aspect of the results of testing various routines is simply this. One may ask 
for an accuracy $\epsilon_{\text {tol }}$. The routine gives a result claiming an accuracy $\epsilon_{\text {est }}$. Checking the exact result reveals that the actual accuracy attained is $\epsilon_{\mathrm{act}}$. These various accuracies stand in ratios such as

$$
\epsilon_{\mathrm{tol}} \cdot \epsilon_{\mathrm{est}} \cdot \epsilon_{\mathrm{act}}=1000: 100: 1 .
$$

Of course it is gratifying to obtain an answer considerably more accurate than required. But it is slightly mortifying to realize that this accuracy has been paid for by using perhaps twice the number of function evaluations necessary.

The iterative method based on successive evaluations of $Q^{(m)} f, m=1,2,4,8 \cdots$, using the convergence criterion (2.36) modified by (2.41) has neither of these undesirable features. All the function evaluations are used in the final result. And one does not have to evaluate $Q^{(2 m)} f$ simply to establish that $Q^{(m)} f$ is sufficiently accurate. In the author's view, it is because of this it is competitive with (but not necessarily better than) the Gauss-Legendre scheme described by Stroud.

We close this section with an example, taken from Stroud [11]. By means of this example

$$
\int_{0}^{\pi} x \cos 3 x d x=-2 / 9
$$

Stroud compares the Romberg integration method [2] with the method suggested by himself using the formulas $G_{4}, G_{6}, G_{8}, \cdots$. In Table 1 we present some of the results of a wider comparison, using other quadrature schemes as well.

\begin{tabular}{|c|c|c|c|c|c|c|c|}
\hline \multirow[b]{2}{*}{$\epsilon_{\text {tol }}$} & & \multicolumn{6}{|c|}{ Routine } \\
\hline & & $\mathrm{l}$ & $\mathrm{H}$ & & ASM & $\mathrm{CQ}^{* *}$ & GS \\
\hline \multirow[t]{3}{*}{$10^{-5}$} & $N$ & (6i) & 65 & & 163 & $1 \% C+3 R$ & 28 \\
\hline & $\epsilon_{\text {est }}$ & $1.2 \times 10^{-6}$ & $3.2 \times 10^{-6}$ & & - & $4.1 \times 10^{-8}$ & $7.8 \times 10^{-7}$ \\
\hline & $\epsilon_{\mathrm{act}}$ & $9.0 \times 10^{-10}$ & $2.4 \times 10^{-8}$ & 4.2 & $\times 10^{-8}$ & $6.1 \times 10^{-9}$ & $5 \times 10^{-10}$ \\
\hline \multirow[t]{3}{*}{$10^{-i}$} & $N$ & 129 & 129 & & 451 & $1 \bar{i} C+3 R$ & 40 \\
\hline & $\epsilon_{\mathrm{est}}$ & $8.1 \times 10^{-10}$ & $4.8 \times 10^{-8}$ & & & $4.1 \times 10^{-8}$ & $3.1 \times 10^{-10}$ \\
\hline & $\epsilon_{\mathrm{act}}$ & $9.0 \times 10^{-11}$ & $2.7 \times 10^{-10}$ & 2.9 & $\times 10^{-9}$ & $6.1 \times 10^{-9}$ & $1.9 \times 10^{-10}$ \\
\hline \multirow[t]{3}{*}{$10^{-9}$} & $N$ & 129 & 129 & & 1351 & $31 C+5 R$ & 40 \\
\hline & $\epsilon_{\text {est }}$ & $8.1 \times 10^{-10}$ & $5.9 \times 10^{-10}$ & & - & $9.1 \times 10^{-10 *}$ & $3.1 \times 10^{-10}$ \\
\hline & $\epsilon_{\text {act }}$ & $9.0 \times 10^{-11}$ & $9.8 \times 10^{-11}$ & 1.9 & $\times 10^{-10}$ & $1.0 \times 10^{-10}$ & $1.9 \times 10^{-10}$ \\
\hline
\end{tabular}

Table 1

The machine used has a machine accuracy parameter $\epsilon_{m}=0.75 \times 10^{-11} ; K_{f}=4.0$.

* This is actually an estimate, given by the routine of the round-off level. Intermediate output indicated that, without this precaution $\epsilon_{\mathrm{est}}=3.2 \times 10^{-10}$, an estimate contaminated by round-off error.

** The notation $N_{1} C+N_{2} R$ stands for $N_{1}$ function evaluations with complex argument and $\mathrm{N}_{2}$ function evaluations with real argument.

The results obtained using several standard quadrature routines are listed.

R....... Romberg Integration [2], [1].

H....... Romberg Integration as adapted by Håvie [6].

ASM...... Adaptive Simpson [7] using Modification [8].

GS........ The Gauss Legendre Rules following a method suggested by Stroud [11].

CQ........ Complex Quadrature Scheme described in this paper. 
An interesting feature of this example is the effect of round-off error. The machine accuracy parameter here is

$$
\epsilon_{m}=0.75 \times 10^{-11} \text {. }
$$

By inspection one sees that (taking $K_{f}=4$ ) the effective round-off level for routines using only real function evaluations is

$$
\epsilon_{\mathrm{r} \cdot \mathrm{o}}=4 \pi \epsilon_{m} \times \max _{0<x<\pi}|f(x)|=2.0 \times 10^{-10} .
$$

In fact, none of these quadrature routines contained any round-off error failure warning and if a lower value of $\epsilon_{\text {tol }}$ is required sometimes a very large amount of work (to the physical limits of the routine) is carried out. The complex quadrature routine becomes aware of trouble of this nature at an early stage. Since the maximum of $|f(z)|$ within any region of the complex plane occurs on the boundary, the relevant level is higher. In fact

$$
\epsilon_{\mathrm{r} \cdot \mathrm{o}}=4 \pi \epsilon_{m} \times \max _{|z-\pi / 2|=\pi / 2}|f(z)|=1.0 \times 10^{-8} .
$$

In the case in which $\epsilon_{\text {tol }}=10^{-9}$, the routine found, after one complex and three real function evaluations,

$$
\epsilon_{\mathrm{r} \cdot \circ}^{(2)}=4 \pi \epsilon_{m} \times \max _{j=1,4}\left|f\left(\frac{\pi}{2}+\frac{\pi}{2} e^{\pi i j / 2}\right)\right|=0.9 \times 10^{-8} .
$$

Consequently, at this relatively early stage, this contour was abandoned. The integration interval was divided into two equal sections and each section was successfully treated separately. In this way the total number of function evaluations used was $31 \mathrm{C}+5 \mathrm{R}$, and only one of these evaluations was not used in the final result.

The early recognition of the presence and magnitude of round-off error is a desirable feature of this method.

In a series of other examples, the author has found that in general the number of complex function evaluations required to obtain a particular accuracy is less than the number of real function evaluations required by the Gauss-Legendre scheme used here, but greater than half this number. Almost invariably the number of function evaluations required by the other routines is much larger.

To sum up, the disadvantages of the suggested method include the restriction to analytic functions with no close singularities and the necessity of using complex function evaluations. Moreover the results are marginally inferior to a GaussLegendre scheme, but significantly superior to other standard schemes. An additional by-product is a set of Taylor coefficients which represent the function within a circle having the interval of integration as diameter.

4. Quadrature with Variable Limits. The method described in the previous section may be adapted without difficulty to the case in which several integrals having the same integrand but different limits are required. That is to say we require approximations to

$$
I[A, B] f(x)=\int_{A}^{B} f(x) d x=F(B)-F(A)
$$


for a set of values of $B$ and $A$. So long as we may choose a circle of radius $r$ in which $f(z)$ is analytic and which contains all the required values of $A$ and $B$, i.e.,

$$
-r \leqq A<B \leqq r
$$

it follows that

$$
F(x)=x \sum_{s=0}^{\infty} \frac{r^{s} a_{s}}{s+1}\left(\frac{x}{r}\right)^{s}
$$

This may be approximated by

$$
F^{[m]}(x)=x \sum_{s=0}^{m-1} \frac{r^{s} a_{s}{ }^{(m)}}{s+1}\left(\frac{x}{r}\right)^{s}
$$

and, following the same reasoning as in the previous section, we have

$$
\left|F(x)-F^{[m]}(x)\right|<\frac{x(I(R))^{1 / 2}}{1-x / R} \rho^{m}
$$

where as before

$$
\rho=r / R<1
$$

and $I(R)$ is given by $(2.26)$.

Such a method might be used to tabulate $I\left[-r, B_{i}\right]$ at equal intervals

$$
B_{i}=-r+i r / n, \quad i=1,2, \cdots, 2 n .
$$

A more interesting application where the method appears to advantage is in a problem involving interpolation with a function defined as an integral. For example, if one wishes to solve for $y$ the equation

$$
\int_{-r}^{y} f(x) d x=b g(y)
$$

where there is a solution satisfying $0<y<r$, the accuracy to which the integral is required depends on both the accuracy to which the solution $y$ is required and the behavior of $f(x)$ in the neighbourhood of $x=y$. In this case one would naturally use the Lagrange scheme or the Newton-Raphson scheme to look for the zero of an approximate function

$$
\phi^{[m]}(y)=F^{[m]}(y)-F^{[m]}(-r)-b g(y)
$$

using initially a relatively small value of $m$. Approximations to $\phi^{[m]^{\prime}}(y)$ and $\phi^{[m]^{\prime \prime}}(y)$ if required may be based on the same set of Taylor coefficients. Since a reliable error estimate for the difference $F^{[m]}(y)-F(y)$ is available it is quite a simple matter to construct a scheme by which if necessary the value of $m$ is raised to its proper level only towards the end of the calculation when the required accuracy in $F^{[m]}(y)$ is more completely known.

5. Quadratures with Weighting Functions $x^{n} \ln |x|$ and $|x|^{\alpha}$. A very convenient application of the above theory is the calculation of integrals of the form

$$
I[A, B](w(x) f(x))=\int_{A}^{B} w(x) f(x) d x=F(B)-F(A) .
$$


Here $f(x)$ is as usual an analytic function whose radius of convergence $R_{c}$ exceeds $r$ and

$$
-r \leqq A<B \leqq r .
$$

The function $w(x)$ on the other hand need not be analytic, but the indefinite integrals

$$
\int^{x} w(y) y^{s} d y=V_{s}(x)
$$

should have a simple form. We consider here only the examples

$$
\begin{aligned}
& w(x)=|x|^{\alpha} \\
& w(x)=x^{n} \ln |x| .
\end{aligned}
$$

Naturally if the singularity at $x=0$ lies within the interval of integration, we have to restrict $\alpha$ or $n$ so that the integral exists, i.e.

$$
\begin{aligned}
& \alpha>-1, \quad(A B \leqq 0), \\
& n \geqq 0, \quad(A B \leqq 0) .
\end{aligned}
$$

In general, it is convenient to express $F(x)$ in the form

$$
F(x)=d(x) \sum_{s=0}^{\infty} C_{s}\left(r^{s} a_{s}\right)\left(\frac{x}{r}\right)^{s}
$$

where

$$
\begin{aligned}
w(x)=|x|^{\alpha} ; \quad C_{s} & =1 /(s+\alpha+1), \quad s \neq-\alpha-1, \\
d(x) & =x|x|^{\alpha}, \quad s \neq-\alpha-1, \\
d(x) C_{s} & =x|x|^{\alpha} \ln |x|, \quad s=-\alpha-1, \\
w(x)=|x|^{n} \ln |x| ; C_{s} & =\frac{\ln |x|}{s+n+1}-\frac{1}{(s+n+1)^{2}}, \quad s \neq-n-1 \\
d(x) & =x|x|^{n}, \quad s \neq-n-1, \\
d(x) C_{s} & =x|x|^{n} \frac{1}{2}\{\ln |x|\}^{2}, \quad s=-n-1 .
\end{aligned}
$$

The distinction between $d(x)$ and $C_{s}$ is arbitrary here. The above choice is convenient for coding the problem except in the particular case $s=-\alpha-1$ or $s=$ $-n-1$ which has to be dealt with specially in an automatic code.

An approximation to (5.8), based on $m$ function values on the circle $|z|=r$ and the calculation of approximations $r^{s} a_{s}{ }^{(m)}, s=0,1, \cdots, m-1$ to the Taylor coefficients described in Section 2, is

$$
F^{[m]}(x)=d(x) \sum_{s=0}^{m-1} C_{s}\left(r^{s} a_{s}^{(m)}\right)\left(\frac{x}{r}\right)^{s} .
$$

Following precisely the method of Section 3, we find the difference 


$$
F^{\prime}(x)-F^{[m]}(x)=d(x) \sum_{s=m}^{\infty} r^{s} a_{s}\left\{C_{s}\left(\frac{x}{r}\right)^{s}-C_{s(\bmod m)}\left(\frac{x}{r}\right)^{s(\bmod m)}\right\}
$$

and the magnitude is bounded by

$$
\left|F(x)-F^{[m]}(x)\right|<d(x) e(I(R))^{1 / 2} \rho^{m} /(1-\rho)
$$

where $I(R)$ and $\rho$ have their usual meanings and $e$ is a convenient constant satisfying

$$
e>\sup _{s \geqq m}\left\{C_{s}\left(\frac{x}{r}\right)^{s}-C_{s(\bmod m)}\left(\frac{x}{r}\right)^{s(\bmod m)}\right\} .
$$

In the cases in which the singularity is integrable, convenient values of $e$ are

$$
\begin{aligned}
& e=1 /(\alpha+1), \quad \alpha>-1, \\
& e=\left|\frac{|\ln | x||}{n+1}+\frac{1}{(n+1)^{2}}\right|, \quad n \geqq 0 .
\end{aligned}
$$

Cases in which these methods appear to advantage include the problem of quadrature close to an unintegrable singularity. It is convenient to discuss this by means of an example. We consider the numerical evaluation of

$$
\int_{\Lambda}^{1 / 2} \frac{1}{\sin ^{2} \pi x} d x=F(1 / 2)-F(A), \quad A>0 .
$$

Here the exact result is known to be

$$
F(x)=1 / \pi \tan \pi x
$$

and the function $1 / \sin ^{2} \pi x$ has a pole of order 2 at the origin. We are interested in obtaining results for various values of $A$ some of which are relatively small, the smallest being $A_{\min }$. We require a preset accuracy $\epsilon_{\mathrm{tol}}$.

We write the integral in the form

$$
\left[[A, 1 / 2]\left(f(x) x^{-2}\right)=\int_{A}^{1 / 2} \frac{f(x)}{x^{2}} d x\right.
$$

where

$$
f(z)=\frac{z^{2}}{\sin ^{2} \pi z}=\sum_{s=0}^{\infty} a_{s} z^{*}, \quad|z|<1
$$

is analytic within the circle $|z|=\frac{1}{2}$. In order to use the convergence criterion, we have to calculate $f(0)$ analy tically

$$
f(0)=\lim _{z \rightarrow 0} \frac{z^{2}}{\sin ^{2} \pi z}=\frac{1}{\pi^{2}} .
$$

The exact formula is

$$
F(A)=-\frac{1}{A}\left\{-a_{0}+\left(a_{1} r\right) \frac{A}{r} \ln |A|+\sum_{s=2}^{\infty} \frac{r^{s} a_{s}}{s-1}\left(\frac{A}{r}\right)^{s}\right\}
$$

and the approximation based on $m$ function values is 


$$
F^{[m]}(A)=-\frac{1}{A}\left\{-a_{0}{ }^{(m)}+\left(r a_{1}{ }^{(m)}\right) \frac{A}{r} \ln |A|+\sum_{s=2}^{m-1} \frac{r^{s} a_{s}{ }^{(m)}}{s-1}\left(\frac{A}{r}\right)^{s}\right\}
$$

where $r=\frac{1}{2}$. The accuracy to which the Taylor coefficients are required to give an accuracy of $\epsilon_{\mathrm{tol}} / 2$ for $F^{[m]}(A)$ is estimated as $\epsilon_{\mathrm{tol}} / 2 A$. Thus if $A_{\min }$ is very close to zero, excessive accuracy may be required for these coefficients. In Table 2 we present a selection of the results of an automatic code written to evaluate this integral. The accuracy in $I\left[A_{\mathrm{min}}, 1 / 2\right] w(x) f(x)$ as estimated by the routine and the actual accuracy attained are given, together with the parameter $m$. The number $m$ is not in this case the actual number of function evaluations. In general if $f(x)$ is real, only $m / 2-1$ complex function evaluations and three real function evaluations are required. In this particular example (which is exceptional) the fact that $f(z)$ is even in $z$ may be exploited to reduce this number further to $m / 4$ complex and two real. Based on the same set of function evaluations approximations to $I\left[A, \frac{1}{2}\right] w(x) f(x)$ for values of $A$ between $A_{\text {min }}$ and $\frac{1}{2}$ were also obtained; these results, not given here, all gave more accurate results and correspondingly closer estimates of their accuracy.

This example is interesting because it was used by Eisner [4] to illustrate a method for integrating near a pole. Subsequently it became the subject of correspondence, Squire [10] suggesting that a classical method is perhaps more suitable, and Eisner [5] remaining unconvinced. Some of the results obtained by Eisner and by Squire are included in the table. A comparison between either of these schemes and the approach suggested here indicates the sort of advantages which accrue as a result of accepting the initial inconvenience of allowing complex function values.

In the method presented here the user asks for a particular accuracy $\epsilon_{\text {tol }}$ and the routine provides a result with an accuracy estimate $\epsilon_{\text {est }}$. If subsequently a more accurate result is required the calculation may be taken up at the point at which it was discontinued. However, if higher accuracy is not attainable because of roundoff error, this is indicated. In fact the routine indicates its estimate of the round-off error limit in any case.

Standard methods could be quite easily modified to do the same thing, but only at a significant cost in terms of function evaluations.

A more interesting difference concerns the actual function values used. It appears that if an integration near a singularity is to be effected and function values are restricted to the real line, then some at least of these function values are required close to the singularity. Thus Eisner's method relies on function evaluations of $1 / \sin ^{2} \pi x$ at $x=A_{\min }, 2 A_{\text {min }} \cdots$ and Squire's approach requires function evaluations of

$$
\left(\frac{1}{\sin ^{2} \pi x}-\frac{1}{\pi^{2} x^{2}}\right)
$$

at values of $x$ some of which are close to $A_{\min }$. Since $A_{\min }$ is small, care has to be taken to avoid errors of a numerical nature in any of these critical function evaluations. On the other hand, the method given here requires function evaluations of $z^{2} / \sin ^{2} \pi z$ on the circle $|z|=\frac{1}{2}$ where there are no difficulties of that type. It does require also the value $f(0)$ in order to apply the convergence criterion. (Eisner's method does not require this and Squire's method requires both $f(0)$ and $f^{\prime}(0)$.) The 
large value of the integral comes about, not as a consequence of large function values, but because of the overall scaling factor $1 / A_{\min }$ in (5.22).

The table indicates that using $m=64$, in this exceptional case 16 complex function evaluations and 2 real function evaluations, a machine accuracy result is obtained however close to the singularity one might wish to integrate.

We close this section with the remark that Gaussian methods, specially designed for the particular singularity in question, might well be superior to the method described here, the comparison possibly being of the same nature as in the quadrature described in Section 3. However, to the author's knowledge Gaussian methods for functions with singularities outside the range of integration are not at present readily available.

TABLE 2

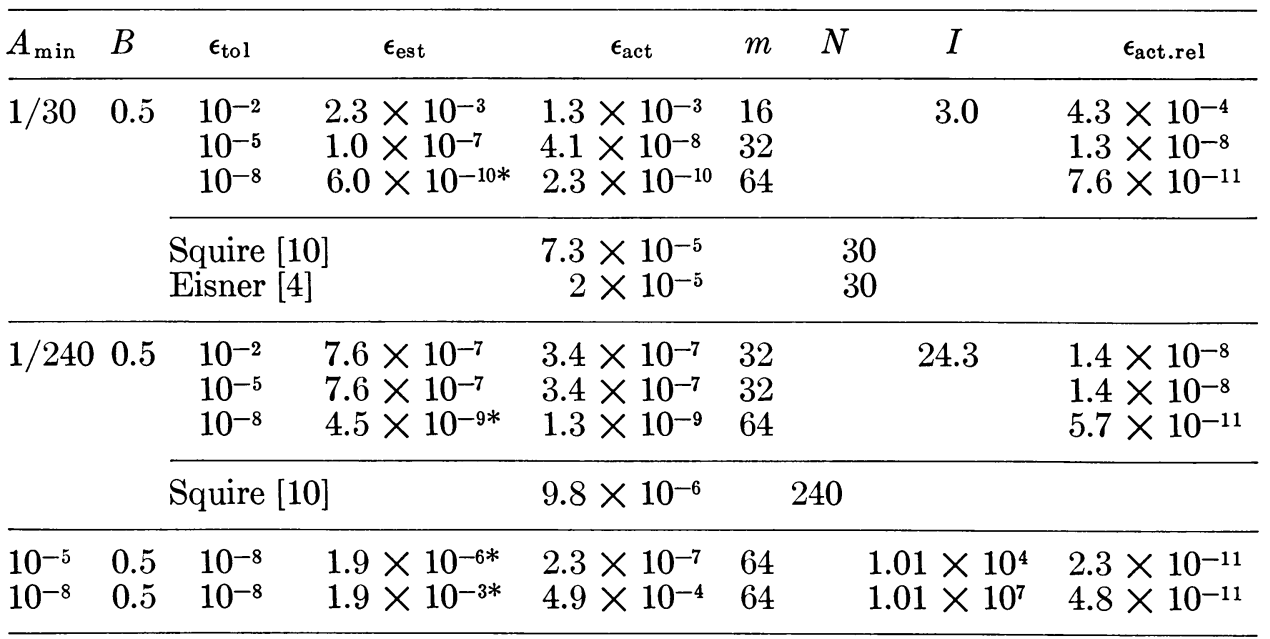

* This result is an estimate based on round-off level; $\epsilon_{m}=0.75 \times 10^{-11} ; K_{f}=10$.

The meanings of columns 1-6 are explained in the text.

$N$ is number of function values.

$I$ is the true $I\left[A_{\min }, B\right] \operatorname{cosec}^{2} \pi x$.

$\epsilon_{\text {act.rel }}$ is the actual relative error of the result.

All the results here (other than those due to Eisner and Squire) are obtained on the basis of the same $16 \mathrm{C}+2 \mathrm{R}$ function values $z^{2} / \sin ^{2} \pi z$.

6. Subdivision of Interval. One of the important aspects of the method just described is that it can be used with confidence only if the function $f(z)$ has no singularities within the circle $C_{r}|z|=r$. If there are singularities outside this circle, but close to it, the method works but inefficiently. If there are singularities within the circle an alarming feature of this method is that in general it converges, but to an incorrect answer. A saving grace is that in this case $a_{0}{ }^{(m)}$ also in general converges to some value other than $f(0)$ and so there would be some warning about such a failure.

While in general it is an easy matter to locate the singularities of an analytic function, circumstances may arise in which one knows the function is analytic, but does not know the locations of the singularities. An example might be that $f(z)$ is the 
lowest eigenvalue of an equation which is analytic and has $z$ as a parameter. In such cases the user is warned to proceed with extreme caution.

If the locations of the singularities are known, and some do lie within the circle $|z|=r$, the method described here can be used only if the interval of integration is first subdivided, each subinterval having the property that the circle of which it is a diameter contains no singularities. At first sight this might appear to be a major defect of this method, since such a subdivision is not required by standard quadrature schemes. However, it is a matter of experience that while under these circumstances such schemes may be used directly, it is as a rule more efficient to subdivide the interval first in any case. Thus the defect is only minor in the sense that what is expedient in general is mandatory here.

The results of an investigation into the appropriate subdivision method are given here. This investigation is semiheuristic in nature but supported by empirical results.

The singularities of $f(z)$ lie at $\alpha_{1}, \alpha_{2}, \cdots, \alpha_{k}$. The interval $[-r, r]$ is divided into $n$ intervals by

$$
-r=d_{0}<d_{1}<\cdots<d_{n-1}<d_{n}=r
$$

having centers

$$
c_{i}=\left(d_{i}+d_{i-1}\right) / 2, \quad i=1,2, \cdots, n
$$

and lengths $2 r_{i}$, given by $r_{i}=\left(d_{i}-d_{i-1}\right) / 2$. If the singularity closest to $c_{i}$ is a distance $b_{i}$ from $c_{i}$, i.e.,

$$
b_{i}=\min _{j=1, k}\left|c_{i}-\alpha_{i}\right|
$$

it has been found that the total number of function evaluations required is strongly dependent on the set of ratios $b_{i} / r_{i}, i=1, \cdots, n$. The effect of the restriction that the number of function values on any circle has to be a power of two has the effect of introducing a fluctuation which is difficult to predict; the general situation is that the optimum choice is $b_{i} / r_{i}=e=2.71$, but choices involving $2<b_{i} / r_{i}<4$ are at most marginally inferior.

Such a subdivision may usually be constructed by inspection.

7. Conclusions. In this paper a very simple approach to various quadrature problems has been investigated. This approach is merely to express the problem in terms of a Taylor series and to carry out term by term integration as appropriate. The severe limitation is that the integrand (apart from a weighting function) has to be analytic and complex arithmetic is involved. The emphasis of this paper is not on the methods (which involve little more than freshman calculus) but on the estimation and control of the accuracy and the recognition and control of round-off error.

It appears to be the case that in problems which have been extensively analyzed, such as quadrature between fixed limits (Section 3), the most sophisticated methods available are better than the one presented here, though not by a very wide margin. However, this method is superior to some other standard routines.

On the other hand in problems which at this time have not received any thorough 
treatment from numerical analysts, such as the problem described in Section 5 , the method presented here is clearly a considerable improvement on the few standard techniques available.

The author feels that there may be many other problems in which an approach of this type might be rewarding, but of course only problems which principally involve analytic functions are susceptible to this sort of treatment.

Argonne National Laboratory

Applied Mathematics Division

Argonne, Illinois 60439

1. F. L. Baule, "Algorithm 60; Romberg Integration," Comm. Assoc. Comput. Mach., v. 4, 1961 , p. 255 ; ibid., v. 5, 1962, p. 168 .

2. F. L. Baule, H. Rutishauser \& E. Stiefel, "New aspects in numerical quadrature," Proc. Sympos. Appl. Math., Vol. 15, Amer. Math. Soc., Providence, R. I., 1963, pp. 199-218. MR 30 \#4384.

3. G. BirкноFF \& D. M. Young, "Numerical quadrature of analytic and harmonic functions," J. Mathematical Phys., v. 29, 1950, pp. 217-221.

4. E. Eisner, "Numerical integration of a function that has a pole," Comm. Assoc. Comput. Mach., v. 10, 1967, pp. 239-243.

5. E. Eisner, "On numerical integration of a function that has a pole," Comm. Assoc. Comput. Mach., v. 10, 1967, p. 610 .

6. T. Hivik, "On a modification of Romberg's algorithm," Nordisk Tidskr. InformationsBehandling, v. 6, 1966, pp. 24-30. MR $33 \# 3460$.

7. W. M. McKeEman, "Algorithm 145; adaptive numerical integration by Simpson's rule," Comm. Assoc. Comput. Mach., v. 5, 1962, p. 604.

8. W. M. McKerman, "Certification of algorithm 145; adaptive numerical integration by Simpson's rule," Comm. A ssoc. Comput. Mach., v. 6, 1963, pp. 167-168.

9. J. MCNAMEE, "Error-bounds for the evaluation of integrals by the Euler-Maclaurin formula and by Gauss-type formulae," Math. Comp., v. 18, 1964, pp. 368-381. MR 32 \#3264.

10. W. SQuire, "On numerical integration of a function that has a pole," Comm. Assoc. Comput. Mach., v. 10, 1967, p. 610.

11. A. H. S'rroud, "Error estimates for Romberg quadrature," J. Soc. Indust. Appl. Math. Ser. B Numer. Anal., v. 2, 1965, pp. 480-488. MR $34 \# 957$.

12. P. J. Davis \& P. Rabinowitz, Numerical Integration, Blaisdell, Waltham, Mass., 1967. MR $35 \# 2482$.

13. V. I. Krylov, Approximate Calculation of Integrals, Fizmatgiz, Moscow, 19:59; 2nd ed., 1966; English transl., Macmillan, New York, 1962; 1966. MR 22 \#2002; MR $26 \# 2008$. 